\title{
Computing interior transmission eigenvalues for homogeneous and anisotropic media
}

\author{
Andreas Kleefeld and Lukas Pieronek $\ddagger$ \\ Forschungszentrum Jülich GmbH, Jülich Supercomputing Centre, 52425 Jülich, \\ Germany \\ E-mail: a.kleefeld@fz-juelich.de and l.pieronek@fz-juelich.de \\ Accepted at Inverse Problems; Preprint
}

\begin{abstract}
The method of fundamental solutions is investigated in a stabilized version for the computation of interior transmission eigenvalues in two dimensions for homogeneous and anisotropic media without voids. This approach has already proven to be very competitive in practice for the isotropic framework among regular scattering shapes and keeps predominating through its simplicity as being mesh- and integration free. We give a new approximation analysis, present various numerical results and show that the eigenvalue spectrum for isotropic scatterers is generally different from the corresponding anisotropic borderline cases.
\end{abstract}

Keywords: interior transmission eigenvalues, method of fundamental solutions, anisotropic scattering

\section{Introduction}

Interior transmission eigenvalues (ITEs) arise necessarily in the study of inverse scattering problems as a criterion for exclusion to make conventional qualitative methods work - at least from a theoretical perspective, see [19, 5]. As such, on the one hand they are impractical for interactive experiments with penetrable (anisotropic) media, see $[6,18,17]$, but on the other hand their individual magnitudes themselves also encode basic information about the scatterer's interior and thus exhibit applications in nondestructive testing, cf. [8, 14]. For both purposes it is crucial to understand their mathematical structure and to be able to compute them with high accuracy. Since the latter seems to lag a little behind the theory for homogeneous and anisotropic media, this paper is to present a relatively easy algorithm for the calculation of corresponding ITEs in 2D.

Our incorporated technique is a synthesis of the method of fundamental solutions (MFS) and a stabilization scheme as first presented in [2] which together result $\ddagger$ Author to whom any correspondence should be addressed. 
in a robust Trefftz collocation method. Other currently used approaches for the computation of anisotropic ITEs are usually executed by the boundary element method, see $[23,20,15]$, or by the finite element method, see $[9,12,16,15]$, which are as such not meshless. Despite its simplicity, our advanced MFS version has already proven to be a great success for many ordinary but isotropic scatterers, see [21], and can still provide very accurate results in the anisotropic case, offering a partial alternative for the open conclusion in [9]. Further features to be optionally adopted within our proposed

algorithm are the recovery of the eigenvalue's multiplicity from the discretized setting as well as their approximate eigenfunctions that can be alternatively used to investigate local properties similar to [3].

Although almost identical in the numerical implementation, the underlying eigenproblem associated with ITEs, the interior transmission problem (ITP), classifies as a non-selfadjoint and non-elliptic PDE system and fundamentally distinguishes between the isotropic and the anisotropic scenario through the required regularity assumptions of the assigned eigenfunctions. This calls for an individual approximation theory compared to [21] and is therefore analyzed below for two of the theoretically most investigated anisotropy classes. For the first time, our numerical results then also show that in both regimes the corresponding borderline ITEs behave completely different even though the constitutive parameters approach the same isotropic setup. A final excursion for the disc as representative scatterer then reveals the nature of this erratic behavior in that special case.

The remainder of this paper is structured as follows: In section 2 we will recall the ITP and introduce the general MFS setup for computing ITEs including its practical implementation. We investigate the approximation aspects for our method from the abstract ITP function space perspective in section 3 and present then our computational results for a variety of scattering objects in section 4 where we focus on four different anisotropic cases. A final conclusion will summarize the pros and cons of our proposed algorithm.

\section{Problem setup}

\subsection{Modeling the homogeneous and anisotropic ITP}

Let the penetrable scatterer $D$ be mathematically represented by a simply connected and bounded region in 2D with Lipschitz boundary $\partial D$. Further we denote the constant index of refraction by $n>0$ whose anisotropic structure is reflected by the symmetric positive definite matrix $A \in \mathbb{R}^{2 \times 2}$. Its eigenvalues

$$
A_{*}:=\min _{\substack{\xi \in \mathbb{R}^{2} \\|\xi|=1}}(\xi \cdot A \xi), \quad A^{*}:=\max _{\substack{\xi \in \mathbb{R}^{2} \\|\xi|=1}}(\xi \cdot A \xi)
$$


are assumed to satisfy either $A_{*}>1$ or $A^{*}<1$. The ITP for anisotropic media then reads: Find $k \in \mathbb{C} \backslash\{0\}$ such that there exist non-trivial solutions $v, w \in H^{1}(D)$ to

$$
\begin{aligned}
\operatorname{div}(A \nabla w)+k^{2} n w=0 & \text { in } D, \\
\Delta v+k^{2} v=0 & \text { in } D, \\
w=v & \text { on } \partial D, \\
\partial_{\nu_{A}} w=\partial_{\nu} v & \text { on } \partial D .
\end{aligned}
$$

In this case, $k$ is called an ITE. Here $\partial_{\nu_{S}} \bullet:=\nu \cdot(S \nabla \bullet)$ denotes the co-normal derivative for any symmetric $S \in \mathbb{R}^{2 \times 2}$. The system (1) emerges, for example, as a special case of the 3D time-harmonic electromagnetic scattering problem if $D$ has planar symmetry which then reduces the setup to our scalar Helmholtz case for the propagating field components, see $[9,7]$. Note the improved Sobolev regularity assumptions of $v, w$ separately in comparison with the ITP for isotropic media which is now due to the perturbation of $w$ in the highest order Laplacian term by the anisotropy $A$. Since the resulting PDE system generally prevents a transformation into a fourth order elliptic equation then, the eigenproblem analysis requires a different, variational approach for which $H^{1}(D)$ turns out to provide the conventional Fredholm framework again with its typical existence and uniqueness results under the above distinction of $A_{*}$ and $A^{*}$, see $[10,22]$. It reads for the ITP in (1)

$$
\int_{D}-A \nabla w \cdot \nabla \varphi+\nabla v \cdot \nabla \psi+k^{2} n w \varphi-k^{2} v \psi \mathrm{d} x=0
$$

where $\varphi, \psi \in H^{1}(D)$ are test functions such that $(\varphi-\psi) \in H_{0}^{1}(D)$. In what follows, we try to find approximations of ITEs $k$ by seeking for a sequence of approximate eigenfunctions solving the above equation modulo boundary terms which should vanish in some limiting procedure.

\subsection{Solving the ITP: MFS vs. modified MFS}

We apply the MFS, sometimes called the method of auxiliary sources or charge simulation method, for the computation of approximate ITEs as already approved in the context of isotropic media, see [21]. Given the ITP with constant constitutive coefficients $n, A$ and the generic positive wave number $\kappa$ to represent $k$ in (1), the main idea of the MFS is to look for radial basis functions divided into

$$
V_{m}=\left\{\varphi_{1}, \ldots, \varphi_{m}\right\}, \quad W_{m}=\left\{\psi_{1}, \ldots, \psi_{m}\right\}
$$

which solve the given PDE for $v$ and $w$ from the ITP exactly, but generally not the prescribed boundary data yet, respectively. Specific to the MFS is the particular choice of the trial functions $\varphi_{j}(\cdot)=\Phi_{\kappa}\left(\cdot, y_{j}\right)$ and $\psi_{j}(\cdot)=\Psi_{\kappa}\left(\cdot, y_{j}\right)$ which are based upon the corresponding fundamental solution $\Phi_{\kappa}$ or $\Psi_{\kappa}$ of the underlying PDE in (1). More precisely, since two variables are associated with each fundamental solution in which 
it is symmetric, we will use the first one as the functional argument on $D$ to fulfill the constraining PDE and freeze the second one as a parameter to generate linear independent solutions. For the latter we take some fixed exterior contour $\Gamma$, also called the artificial (source) boundary and which must lie disjoint from $\bar{D}$ to make collocation work without inner singularities. Then we pick $m$ distinct source points $\left\{y_{j}\right\}_{1 \leq j \leq m} \subset \Gamma$ which thus determine the dimension of both $V_{m}$ and $W_{m}$. By linearity of the PDEs involved, we can approximate our eigenfunctions now by proper linear combinations

$$
v_{m}(\cdot)=\sum_{j=1}^{m} c_{\Phi}\left(y_{j}\right) \Phi_{\kappa}\left(\cdot, y_{j}\right), \quad w_{m}(\cdot)=\sum_{j=1}^{m} c_{\Psi}\left(y_{j}\right) \Psi_{\kappa}\left(\cdot, y_{j}\right)
$$

and, to prepare for a more compact matrix notation, we define the associated coefficient vector $c_{m} \in \mathbb{C}^{2 m}$ by

$$
c_{m}=\left(c_{\Phi}\left(y_{1}\right), \ldots, c_{\Phi}\left(y_{m}\right),-c_{\Psi}\left(y_{1}\right), \ldots,-c_{\Psi}\left(y_{m}\right)\right)^{\top},
$$

whose optimal choice in the sense of the MFS we will discuss next.

The canonical MFS tries to determine this coefficient vector by matching $m$ boundary collocation points $\left\{s_{j}\right\}_{1 \leq j \leq m} \subset \partial D$ such that both $v_{m}\left(s_{j}\right)-w_{m}\left(s_{j}\right) \approx 0$ and $\partial_{\nu}\left(v_{m}\left(s_{j}\right)-w_{m}\left(s_{j}\right)\right) \approx 0$ are fulfilled for all $j$. This results in a quadratic linear system which, for the purpose of our actual eigenproblem, might tempt us to call those $\kappa$ an approximate ITE admitting some non-trivial $c_{m}$. To understand why this leads to a numerical pitfall, we identify (2) as discrete realizations of the single layer operator given by

$$
\begin{aligned}
& \widehat{S}_{\Phi}(\kappa): c_{\Phi} \mapsto \int_{\Gamma} \Phi_{\kappa}(\cdot, y) c_{\Phi}(y) \mathrm{d} s(y), \\
& \widehat{S}_{\Psi}(\kappa): c_{\Psi} \mapsto \int_{\Gamma} \Psi_{\kappa}(\cdot, y) c_{\Psi}(y) \mathrm{d} s(y),
\end{aligned}
$$

which map continuous coefficient functions $c_{\Phi}, c_{\Psi} \in L^{2}(\Gamma)$ into the space of ITP interior solutions, respectively. Due to compactness of these operators (when their ranges are endowed with any proper Sobolev norm on $\partial D)$, we know that the task of finding $\left(c_{\Phi}, c_{\Psi}\right)$ given any boundary function results in an ill-posed problem for every $\kappa$. Therefore, the standard MFS as the discrete analogon suffers very early from ill-conditioning effects such as spurious eigenvalue detection, see [21].

In 2005, Betcke and Trefethen came up with an effective remedy for a related problem in their paper [2]. They realized that pure boundary collocation calculus ignores any contribution from the eigenfunction's behavior in the domain $D$ itself and thus indirectly supports the ill-posedness of the underlying boundary operator formulation mentioned above. With a trick based on the $Q R$ decomposition, they proposed to add an implicit constraint in form of a relatively large interior norm to the trial functions whose adapted implementation we now summarize for our anisotropic MFS-ITP context and which we will refer to as the modified MFS: 
Sample $m_{I}$ fixed (with respect to $m$ ) interior points $\left\{x_{j}\right\}_{1 \leq j \leq m_{I}} \subset D$ and define the following block matrix associated to our trial functions

$$
T(\kappa)=\left(\begin{array}{cc}
S_{\Phi}(\kappa) & S_{\Psi}(\kappa) \\
\partial_{\nu} S_{\Phi}(\kappa) & \partial_{\nu} S_{\Psi}(\kappa) \\
\widetilde{S}_{\Phi}(\kappa) & 0 \\
\partial_{1} \widetilde{S}_{\Phi}(\kappa) & 0 \\
\partial_{2} \widetilde{S}_{\Phi}(\kappa) & 0 \\
0 & \widetilde{S}_{\Psi}(\kappa) \\
0 & \partial_{1} \widetilde{S}_{\Psi}(\kappa) \\
0 & \partial_{2} \widetilde{S}_{\Psi}(\kappa)
\end{array}\right) \in \mathbb{C}^{\left(2 m+6 m_{I}\right) \times 2 m}
$$

where the upper dense lines correspond to the boundary part from the standard MFS

$$
\begin{gathered}
\left(S_{\Phi}(\kappa)\right)_{i, j}=\Phi_{\kappa}\left(s_{i}, y_{j}\right), \quad 1 \leq i, j \leq m \\
\left(\partial_{\nu} S_{\Phi}(\kappa)\right)_{i, j}=\partial_{\nu} \Phi_{\kappa}\left(s_{i}, y_{j}\right), \quad 1 \leq i, j \leq m
\end{gathered}
$$

and where the diagonal lower part consists of interior point evaluations

$$
\begin{gathered}
\left(\widetilde{S}_{\Phi}(\kappa)\right)_{i, j}=\Phi_{\kappa}\left(x_{i}, y_{j}\right), \quad 1 \leq i \leq m_{I}, 1 \leq j \leq m, \\
\left(\partial_{1} \widetilde{S}_{\Phi}(\kappa)\right)_{i, j}=\partial_{1} \Phi_{\kappa}\left(x_{i}, y_{j}\right), \quad 1 \leq i \leq m_{I}, 1 \leq j \leq m, \\
\left(\partial_{2} \widetilde{S}_{\Phi}(\kappa)\right)_{i, j}=\partial_{2} \Phi_{\kappa}\left(x_{i}, y_{j}\right), \quad 1 \leq i \leq m_{I}, 1 \leq j \leq m .
\end{gathered}
$$

Note that all appearing derivatives on the right-hand sides of our block definitions are understood with respect to the first argument of $\Phi_{\kappa}$. The same notation takes over for $\Phi_{\kappa}$ being replaced by $\Psi_{\kappa}$ analogously. We then perform the celebrated $Q R$ factorization of the system matrix

$$
T(\kappa)=Q(\kappa) R(\kappa)=\left(\begin{array}{l}
Q_{B}(\kappa) \\
Q_{I}(\kappa)
\end{array}\right) R(\kappa), \quad R(\kappa), Q_{B}(\kappa) \in \mathbb{C}^{2 m \times 2 m}
$$

which thus constrains our trial functions to a total norm of 1 as the cumulative contribution of boundary and interior points. The final step is to extract back only the boundary part $Q_{B}$ of the unitary matrix factor and minimize its smallest singular vector locally in $\kappa$, i.e.

$$
\text { Minimize } \quad \kappa \longmapsto \min _{r \in \mathbb{C}^{2 m},|r|=1}\left|Q_{B}(\kappa) r\right|=\left|Q_{B}(\kappa) r_{m}\right| \equiv \sigma_{1}(\kappa), \quad \kappa \in U \subset \mathbb{R}_{>0}
$$

The purpose of the introduction for the auxiliary set $U$ is to separate the detection of distinct eigenvalues. Note that if $\sigma_{1}(\kappa)$ is sufficiently close to zero for some $\kappa=k_{m}$, this indeed indicates the approximation of some real ITE since we have found superposed 
MFS trial functions with small boundary misfit along its $m$ collocation points according to

$$
\sigma_{1}\left(k_{m}\right)=\left|Q_{B}(\kappa) r_{m}\right|=\sqrt{\sum_{i=1}^{m}\left(v_{m}\left(s_{i}\right)-w_{m}\left(s_{i}\right)\right)^{2}+\left(\partial_{\nu}\left(v_{m}\left(s_{i}\right)-w_{m}\left(s_{i}\right)\right)\right)^{2}}
$$

where $\left(v_{m}, w_{m}\right)$ are determined via $r_{m}=R(\kappa) c_{m}$. We then call $k_{m}$ an approximate ITE and $\left(v_{m}, w_{m}\right)$ its approximate eigenfunction pair.

\section{Approximation analysis for ITEs}

The MFS is a powerful tool to approximate solutions of a given eigenproblem with constant coefficients, since the only condition left to control in the computational procedure are the prescribed boundary data. To talk about convergence in terms of (3) and (4), it will be necessary to consider the MFS from a more abstract perspective in which collocation as our numerical indicator for residual quantities is replaced by certain integral norms to be kept small. In order to avoid confusion between the two versions, we will refer to the latter as the continuous MFS.

\subsection{Preliminaries}

The first crucial observation we make is that for constant coefficients both PDEs to be solved within the ITP are Helmholtz equations in disguise. While this assertion is obvious for the component $v$, it also applies for $w$ by a pull-back argument which we will discuss in the following. First recall that $A$ is symmetric positive definite so there also exists a unique symmetric positive definite root $A^{\frac{1}{2}}$ such that $A^{\frac{1}{2}} A^{\frac{1}{2}}=A$. Then we set

$$
D^{A}:=A^{-\frac{1}{2}} D
$$

The following lemma shows that the anisotropic PDE for $w$ solved within the ITP on $D$ is equivalent to a pure Helmholtz equation on $D^{A}$.

Lemma 1. The function $w \in H^{1}(D)$ is a solution of $\operatorname{div}(A \nabla w)+\kappa^{2} n w=0$ in $D$ if and only if its pull-back $w^{A} \in H^{1}\left(D^{A}\right)$ defined by $w^{A}(\cdot):=w\left(A^{\frac{1}{2}} \cdot\right)$ solves $\Delta w^{A}+\kappa^{2} n w^{A}=0$ on $D^{A}$. Likewise, if $\Phi_{\kappa \sqrt{n}}$ is the fundamental solution of the Helmholtz equation with wave number $\kappa \sqrt{n}, \Psi_{\kappa}(x, y):=\operatorname{det} A^{-\frac{1}{2}} \Phi_{\kappa \sqrt{n}}\left(A^{-\frac{1}{2}} x, A^{-\frac{1}{2}} y\right)$ solves $\operatorname{div}\left(A \nabla \Psi_{\kappa}(\cdot, y)\right)+\kappa^{2} n \Psi_{\kappa}(\cdot, y)=\delta_{y}(\cdot)$ in the sense of distributions.

Proof. For the first assertion, one can either exploit elliptic interior regularity and check the identities directly by applying the corresponding second order differential operators pointwise, or one can use the definition of distributional solutions in combination with the transformation formula, which then proves the entire lemma. For the latter define 
the linear mapping $L(x):=A^{-\frac{1}{2}} x$ and associate for any test function $\varphi \in H_{0}^{1}(D)$ its pull-back by $\varphi^{A}:=\varphi \circ L^{-1}$. Then the two PDEs under consideration are connected via

$$
\begin{aligned}
& \int_{D^{A}} \nabla w^{A} \cdot \nabla \varphi^{A}-\kappa^{2} n w^{A} \varphi^{A} \mathrm{~d} x^{A} \\
= & \int_{D^{A}} A^{\frac{1}{2}}\left(\nabla w \circ L^{-1}\right) \cdot \nabla \varphi^{A}-\kappa^{2} n\left(w \circ L^{-1}\right) \varphi^{A} \mathrm{~d} x^{A} \\
= & \operatorname{det} A^{-\frac{1}{2}} \int_{D} A^{\frac{1}{2}} \nabla w \cdot \nabla \varphi^{A} \circ L-\kappa^{2} n w \varphi^{A} \circ L \mathrm{~d} x \\
= & \operatorname{det} A^{-\frac{1}{2}} \int_{D} A^{\frac{1}{2}} \nabla w \cdot A^{\frac{1}{2}} \nabla \varphi-\kappa^{2} n w \varphi \mathrm{d} x \\
= & \operatorname{det} A^{-\frac{1}{2}} \int_{D} A \nabla w \cdot \nabla \varphi-\kappa^{2} n w \varphi \mathrm{d} x .
\end{aligned}
$$

The explicit determination of fundamental solutions for PDEs with varying coefficients might generally require individual and tedious computations. However, the lemma above shows that the $A$-dependent family of equations for the function $w$ can be easily reduced to the natural Helmholtz case with its known fundamental solution indexed by $\kappa$. Therefore we can now specify our ansatz functions from (2): The 2D (radiating) fundamental solution of the Helmholtz equation is given by

$$
\Phi_{\kappa}(x, y)=\frac{\mathrm{i}}{4} H_{0}^{(1)}(\kappa|x-y|)
$$

where $H_{0}^{(1)}$ is the Hankel function of the first kind of order zero. According to the above lemma, we know that $\Psi_{\kappa}$ is given by

$$
\Psi_{\kappa}(x, y):=\operatorname{det} A^{-\frac{1}{2}} \Phi_{\kappa \sqrt{n}}\left(A^{-\frac{1}{2}} x, A^{-\frac{1}{2}} y\right)
$$

\subsection{The anisotropic ITP under continuous measurements}

We adopt the guidelines from [21] to be checked within the following approximation analysis but point out that we now require new techniques to prove the corresponding results in the anisotropic case due to the deviating regularity assumptions of ITP eigenfunctions. According to the MFS setup, $\Phi_{\kappa}$ and $\Psi_{\kappa}$ as given above will generate

our approximation space of eigenfunction candidates. Recall that these trial functions exactly solve the interior but generally not the coupled boundary conditions. So the following inhomogeneous ITP perfectly suits our current situation

$$
\begin{array}{rlrl}
\operatorname{div}(A \nabla \widetilde{w})+\kappa^{2} n \widetilde{w}=0 & & \text { in } D, \\
\Delta \widetilde{v}+\kappa^{2} \widetilde{v}=0 & \text { in } D, \\
\widetilde{v}-\widetilde{w}=\widetilde{f} & \text { on } \partial D, \\
\partial_{\nu} \widetilde{v}-\partial_{\nu_{A}} \widetilde{w}=\widetilde{g} & & \text { on } \partial D .
\end{array}
$$


Since the single layer operators from (3)-(4) restricted to $D$ are produced by smooth kernels, the derived MFS even generates classical solution candidates of the above system in

$$
\mathcal{H}_{A}:=\bigcup_{\kappa \in \mathbb{R}_{>0}} \mathcal{H}_{A}(\kappa)
$$

where

$$
\mathcal{H}_{A}(\kappa):=\left\{(\widetilde{v}, \widetilde{w}) \in C^{\infty}(\bar{D}) \times C^{\infty}(\bar{D}): \operatorname{div}(A \nabla \widetilde{w})+\kappa^{2} n \widetilde{w}=0, \Delta \widetilde{v}+\kappa^{2} \widetilde{v}=0\right\}
$$

The next theorem demonstrates that if we apply the continuous MFS, to be clarified then, with successive output $\left\{k_{m}\right\}_{m \in \mathbb{N}}$ that is assumed to form a Cauchy sequence, their limit point $k$ is indeed a real ITE. In particular, no spurious eigenvalues will arise as accumulation points.

Theorem 2. Assume $\left\{\left(v_{m}, w_{m}, k_{m}\right)\right\}_{m \in \mathbb{N}} \subset \mathcal{H}_{A} \times \mathbb{R}_{>0}$ and $\left\{\left(\theta_{m}^{D}, \theta_{m}^{N}\right)\right\}_{m \in \mathbb{N}} \subset H^{\frac{1}{2}}(\partial D) \times$ $H^{-\frac{1}{2}}(\partial D)$ with the above restrictions on $A$ are such that the following conditions hold:

(i) eigenvalue convergence: $k_{m} \longrightarrow k \neq 0$,

(ii) uniform interior bound: $C^{-1}<\left(\left\|v_{m}\right\|_{H^{1}(D)}^{2}+\left\|w_{m}\right\|_{H^{1}(D)}^{2}\right)<C$ for some $C>1$ and for all $m$ large enough,

(iii) approximate ITP: $(\widetilde{v}, \widetilde{w}, \widetilde{f}, \widetilde{g}, \kappa)=\left(v_{m}, w_{m}, \theta_{m}^{D}, \theta_{m}^{N}, k_{m}\right)$ is a solution of (9) for all $m$ large enough with identical refractive index $n>0$ and asymptotically vanishing boundary data $\left(\left\|\theta_{m}^{D}\right\|_{H^{\frac{1}{2}(\partial D)}}+\left\|\theta_{m}^{N}\right\|_{H^{-\frac{1}{2}(\partial D)}}\right) \longrightarrow 0$ for $m \rightarrow \infty$.

Then, there exists a non-trivial weak solution $(v, w) \in \mathcal{H}_{A}$ of (1) with interior transmission eigenvalue $k$.

Proof. Because of the uniform interior bounds with respect to $H^{1}(D)$ we may apply weak compactness to show that the limit is indeed a non-trivial eigenfunction with ITE $k$. Without relabeling a possibly extracted subsequence, we assume that

$$
v_{m} \rightarrow v \quad \text { in } H^{1}(D), \quad w_{m} \rightarrow w \quad \text { in } H^{1}(D)
$$

Since $v_{m}, w_{m}$ fulfill (9) strongly by assumption, they are also weak solutions of the following variational equation when testing against $\varphi, \psi \in H^{1}(D)$ such that $(\varphi-\psi) \in$ $H_{0}^{1}(D)$. We thus obtain

$$
\int_{D}-A \nabla w_{m} \cdot \nabla \varphi+\nabla v_{m} \cdot \nabla \psi+k_{m}^{2} n w_{m} \varphi-k_{m}^{2} v_{m} \psi \mathrm{d} x=\int_{\partial D}\left(\partial_{\nu_{A}} w_{m}-\partial_{\nu} v_{m}\right) \varphi \mathrm{d} s
$$

and thanks to our asymptotically vanishing boundary data we obtain by the duality product

$$
\int_{\partial D}\left(\partial_{\nu_{A}} w_{m}-\partial_{\nu} v_{m}\right) \varphi \mathrm{d} s \leq\|\varphi\|_{H^{\frac{1}{2}(\partial D)}}\left\|\partial_{\nu_{A}} w_{m}-\partial_{\nu} v_{m}\right\|_{H^{-\frac{1}{2}(\partial D)}}
$$


As the right-hand side of the latter tends to zero for $m \rightarrow \infty$, we see that by $(i)$ the pair $(v, w)$ indeed solves $(1)$ in the weak form

$$
\int_{D}-A \nabla w \cdot \nabla \varphi+\nabla v \cdot \nabla \psi+k^{2} n w \varphi-k^{2} v \psi \mathrm{d} x=0
$$

and further $\partial_{\nu_{A}} w=\partial_{\nu} v$ holds in the sense of $H^{-\frac{1}{2}}$ traces. The fact that $(v-w) \in H_{0}^{1}(D)$ follows from the continuity of the trace operator $\tau: H^{1}(D) \rightarrow H^{\frac{1}{2}}(\partial D)$ and Ker $\tau=$ $H_{0}^{1}(D)$ applied to the bounded sequence $\left\{\left(v_{m}-w_{m}\right)\right\}_{m \in \mathbb{N}} \subset H^{1}(D)$. Thus we are left to prove that $(v, w)$ is non-trivial.

For this purpose we first note that due to our assumptions on $A, k, n$ being realvalued, we may continue the proof with either the real or the imaginary parts of $\left(v_{m}, w_{m}\right)$ depending on which choice still fulfills the uniform lower bound in $(i i)$ infinitely often (with a possibly larger constant $C>1$ ). For simplicity we will not relabel the reduced approximate eigenfunctions in the sequel. Then we observe that if we have a corresponding estimate for the weaker $L^{2}(D)$ norm, i.e.

$$
\liminf _{m \rightarrow \infty}\left(\left\|v_{m}\right\|_{L^{2}(D)}^{2}+\left\|w_{m}\right\|_{L^{2}(D)}^{2}\right)>0
$$

our eigenfunction candidate $(v, w)$ will immediately be non-trivial by its definition as weak $H^{1}(D)$-limit which is compactly embedded in $L^{2}(D)$. Therefore, we assume contrarily that there exists a subsequence, not relabeled, such that

$$
\lim _{m \rightarrow \infty}\left(\left\|v_{m}\right\|_{L^{2}(D)}^{2}+\left\|w_{m}\right\|_{L^{2}(D)}^{2}\right)=0
$$

which implies that our uniform lower bound from $(i i)$ is now completely concentrated on the gradients,

$$
\liminf _{m \rightarrow \infty}\left(\left\|\nabla v_{m}\right\|_{L^{2}(D)}^{2}+\left\|\nabla w_{m}\right\|_{L^{2}(D)}^{2}\right) \geq C>0 .
$$

Since $v_{m}$ is a strong solution of the Helmholtz equation with wave number $k_{m}$, we may use integration by parts against $\psi \in H^{1}(D)$ to obtain

$$
\int_{D} \nabla v_{m} \cdot \nabla \psi \mathrm{d} x=\int_{D} k_{m}^{2} v_{m} \psi \mathrm{d} x+\int_{\partial D} \partial_{\nu} v_{m} \psi \mathrm{d} s
$$

Since the trace operator $\tau: H^{1}(D) \rightarrow H^{\frac{1}{2}}(\partial D)$ is right-invertible, duality shows with $\sup _{m}\left\|v_{m}\right\|_{H^{1}(D)}^{2}<C$ and proper dual elements $\psi$ that $\sup _{m}\left\|\partial_{\nu} v_{m}\right\|_{H^{-\frac{1}{2}}(\partial D)}<\infty$. The same equation but with the choice $\psi=v_{m}-w_{m}=: u_{m}$ therefore also gives

$$
\int_{D} \nabla v_{m} \cdot \nabla u_{m} \mathrm{~d} x=\int_{D} k_{m}^{2} v_{m} u_{m} \mathrm{~d} x+\int_{\partial D} \partial_{\nu} v_{m} u_{m} \mathrm{~d} s
$$

Note that the above right-hand side vanishes for $m \rightarrow \infty$ due to (10) and (iii), so we obtain the relation

$$
\lim _{m \rightarrow \infty} \int_{D} \nabla v_{m} \cdot \nabla u_{m} \mathrm{~d} x=0
$$


Computing ITEs for homogeneous and anisotropic media

For the remainder of the proof we try to find a contradiction with (12) by incorporating our explicit assumptions on the eigenvalues of $A$. First note that $u_{m}$ can also be characterized as a weak solution of the system

$$
\begin{aligned}
\operatorname{div}\left(A \nabla u_{m}\right)+k_{m}^{2} n u_{m}=\operatorname{div}\left((A-I) \nabla v_{m}\right)+k_{m}^{2}(n-1) v_{m} & \text { in } D, \\
u_{m}=\theta_{m}^{D} & \text { on } \partial D, \\
\partial_{\nu_{A}} u=\partial_{\nu_{(A-I)}} v_{m}+\theta_{m}^{N} & \text { on } \partial D
\end{aligned}
$$

whose variational form reads for any $\psi \in H^{1}(D)$

$$
\int_{D}\left(-A \nabla u_{m}+(A-I) \nabla v_{m}\right) \cdot \nabla \psi \mathrm{d} x=\int_{D} k_{m}^{2}\left((n-1) v_{m}-n u_{m}\right) \psi \mathrm{d} x+\int_{\partial_{D}} \theta_{m}^{N} \psi \mathrm{d} s
$$

Assume first that $A_{*}>1$. Choosing $\psi=w_{m}$ in (13) and taking the limit $m \rightarrow \infty$ yields with $(12),(10)$ and some uniform upper bound on $\left\|w_{m}\right\|_{H^{\frac{1}{2}(\partial D)}}$ by continuity of the trace operator

$$
\begin{aligned}
0 & =\lim _{m \rightarrow \infty} \int_{D}\left(-A \nabla u_{m}+(A-I) \nabla v_{m}\right) \cdot \nabla w_{m} \mathrm{~d} x \\
& =\lim _{m \rightarrow \infty} \int_{D}\left(-A \nabla u_{m}+(A-I) \nabla\left(u_{m}+w_{m}\right)\right) \cdot \nabla w_{m} \mathrm{~d} x \\
& =\lim _{m \rightarrow \infty} \int_{D}\left((A-I) \nabla w_{m}-\nabla u_{m}\right) \cdot \nabla w_{m} \mathrm{~d} x \\
& =\lim _{m \rightarrow \infty} \int_{D}(A-I) \nabla w_{m} \cdot \nabla w_{m}-\nabla u_{m} \cdot \nabla\left(v_{m}-u_{m}\right) \mathrm{d} x \\
& =\lim _{m \rightarrow \infty} \int_{D}(A-I) \nabla w_{m} \cdot \nabla w_{m}+\left|\nabla u_{m}\right|^{2} \mathrm{~d} x-\lim _{m \rightarrow \infty} \int_{D} \nabla v_{m} \cdot \nabla u_{m} \mathrm{~d} x \\
& =\lim _{m \rightarrow \infty} \int_{D}(A-I) \nabla w_{m} \cdot \nabla w_{m}+\left|\nabla u_{m}\right|^{2} \mathrm{~d} x \\
& \geq \lim _{m \rightarrow \infty}\left(\left(A_{*}-1\right)\left\|\nabla w_{m}\right\|_{L^{2}(D)}^{2}+\left\|\nabla u_{m}\right\|_{L^{2}(D)}^{2}\right) .
\end{aligned}
$$

Therefore $\nabla w_{m} \rightarrow 0$ in $L^{2}(D)$ and since $\nabla u_{m} \rightarrow 0$ in $L^{2}(D)$ as well, we may conclude

$$
\lim _{m \rightarrow \infty}\left(\left\|\nabla v_{m}\right\|_{L^{2}(D)}^{2}+\left\|\nabla w_{m}\right\|_{L^{2}(D)}^{2}\right)=0
$$

which is a contradiction to (11) in the case $A_{*}>1$. If $A^{*}<1$, we first rewrite (12) in the following way

$$
\begin{aligned}
0 & =\lim _{m \rightarrow \infty}-\int_{D} \nabla v_{m} \cdot \nabla u_{m} \mathrm{~d} x \\
& =\lim _{m \rightarrow \infty} \int_{D}(-A+A-I) \nabla v_{m} \cdot \nabla u_{m} \mathrm{~d} x \\
& =\lim _{m \rightarrow \infty} \int_{D}-A \nabla u_{m} \cdot \nabla v_{m}+(A-I) \nabla v_{m} \cdot \nabla u_{m} \mathrm{~d} x
\end{aligned}
$$


and observe that a combination of (13) with $\psi=v_{m}$ and $\psi=u_{m}$, respectively, yields thanks to (10) and the vanishing boundary data

$$
\begin{aligned}
& \lim _{m \rightarrow \infty} \int_{D}-A \nabla u_{m} \cdot \nabla v_{m}+(A-I) \nabla v_{m} \cdot \nabla u_{m} \mathrm{~d} x \\
= & \lim _{m \rightarrow \infty} \int_{D}(I-A) \nabla v_{m} \cdot \nabla v_{m}+A \nabla u_{m} \cdot \nabla u_{m} \mathrm{~d} x .
\end{aligned}
$$

Putting both equations together gives

$$
\begin{aligned}
0 & =\lim _{m \rightarrow \infty} \int_{D}(I-A) \nabla v_{m} \cdot \nabla v_{m}+A \nabla u_{m} \cdot \nabla u_{m} \mathrm{~d} x \\
& \geq \lim _{m \rightarrow \infty}\left(\left(1-A^{*}\right)\left\|\nabla v_{m}\right\|_{L^{2}(D)}^{2}+A_{*}\left\|\nabla u_{m}\right\|_{L^{2}(D)}^{2}\right) .
\end{aligned}
$$

This again implies by positive definiteness of $A$ that

$$
\lim _{m \rightarrow \infty}\left(\left\|\nabla v_{m}\right\|_{L^{2}(D)}^{2}+\left\|\nabla w_{m}\right\|_{L^{2}(D)}^{2}\right)=0
$$

which also contradicts $(11)$ in the case $A^{*}<1$.

Up to this point we know that the output of the continuous MFS recovers real ITEs $k$ in the limiting process. However, it is not clear yet whether every eigenvalue admits an approximation sequence fulfilling the listed assumptions within Theorem 2 . So the question arises if our trial functions are sufficiently dense. Unlike isotropic media, the definition of ITP eigenfunctions in the anisotropic case allows for interior conditions formulated in the energy space $H^{1}(D)$ for $v$ and $w$ separately which naturally imply the required regularity for the boundary data. In other words, $(v-w)$ is a priori as good as $v$ and $w$ each which directly enables us to consider the approximation problem componentwise. Since by Lemma 1 both interior conditions of the ITP are of Helmholtz type we may restrict our density analysis to the model equation of $v$ first.

Theorem 3. The range of the single layer operator $\widehat{S}_{\Phi}(\kappa)$ from $(3)$ is dense in $\mathcal{A}(\kappa):=\left\{h \in H^{1}(D): \Delta h+\kappa^{2} h=0\right\}$ for all wave numbers $\kappa>0$.

Proof. We aim to show that for fixed $\kappa>0$ the (Hilbert) adjoint of $\widehat{S}_{\Phi}(\kappa)$ given by

$$
\begin{aligned}
\widehat{S}_{\Phi}^{*}(\kappa): \mathcal{A}(\kappa) & \rightarrow L^{2}(\Gamma) \\
h & \mapsto \widehat{S}_{\Phi}^{*}(\kappa) h=\int_{D} \overline{\Phi_{\kappa}(x, \cdot)} h(x)+\nabla_{x} \overline{\Phi_{\kappa}(x, \cdot)} \cdot \nabla h(x) \mathrm{d} x
\end{aligned}
$$

is injective on $\mathcal{A}(\kappa)$ which would then give the desired density result according to the fact that the null space of $\widehat{S}_{\Phi}^{*}(\kappa)$ equals the $H^{1}(D)$-orthogonal complement of the range of $\widehat{S}_{\Phi}(\kappa)$. Hence, we assume that $h \in \mathcal{A}(\kappa)$ is such that $\widehat{S}_{\Phi}^{*}(\kappa) h=0$. This means that the function $\widetilde{h}:=\widehat{S}_{\Phi}^{*}(\kappa) h \in H_{l o c}^{1}\left(\mathbb{R}^{2}\right)$, which extends to $\mathbb{R}^{2}$ naturally as the outcome of a convolution, vanishes along $\Gamma$ and inherits the Sommerfeld radiation condition from $\Phi_{\kappa}$ and $\nabla_{x} \Phi_{\kappa}$. As $\widetilde{h}$ is also a solution of the exterior Helmholtz problem with zero boundary 
data along $\Gamma$, uniqueness and analyticity guarantees that $\widetilde{h}_{\mid D^{c}}=0$ so that we actually identify $\widetilde{h} \in H_{0}^{1}(D)$. This implies on the one hand, since $\bar{h} \in \mathcal{A}(\kappa)$, that

$$
\int_{D}-\nabla \widetilde{h} \cdot \nabla \bar{h}+\kappa^{2} \widetilde{h} \bar{h} \mathrm{~d} x=0
$$

On the other hand, we can find a sequence $\left\{h_{m}\right\}_{m \in \mathbb{N}}$ of smooth and compactly supported functions in $\mathbb{R}^{2}$ such that $h_{m} \rightarrow h$ in $H^{1}(D)$. Now we can compute using integration by parts without boundary contributions as well as the fundamental solution property of the kernel $\Phi_{\kappa}$

$$
\begin{aligned}
& \int_{D}-\nabla \widetilde{h} \cdot \nabla \bar{h}+\kappa^{2} \widetilde{h} \bar{h} \mathrm{~d} x \\
= & \lim _{m \rightarrow \infty} \int_{\mathbb{R}^{2}}-\nabla \widetilde{h} \cdot \nabla \bar{h}_{m}+\kappa^{2} \widetilde{h} \bar{h}_{m} \mathrm{~d} x \\
= & \lim _{m \rightarrow \infty} \int_{\mathbb{R}^{2}} \widetilde{h}\left(\Delta \bar{h}_{m}+\kappa^{2} \bar{h}_{m}\right) \mathrm{d} x \\
= & \lim _{m \rightarrow \infty} \int_{\mathbb{R}^{2}}\left(\int_{D} \overline{\Phi_{\kappa}(y, x)} h(y)+\nabla_{y} \overline{\Phi_{\kappa}(y, x)} \cdot \nabla h(y) \mathrm{d} y\right)\left(\Delta \bar{h}_{m}+\kappa^{2} \bar{h}_{m}\right) \mathrm{d} x \\
= & \lim _{m \rightarrow \infty} \int_{\mathbb{R}^{2}} \int_{D} \overline{\Phi_{\kappa}(y, x)} h(y) \mathrm{d} y\left(\Delta \bar{h}_{m}+\kappa^{2} \bar{h}_{m}\right)-\int_{D} \nabla_{x} \overline{\Phi_{\kappa}(y, x)} \cdot \nabla h(y) \mathrm{d} y\left(\Delta \bar{h}_{m}+\kappa^{2} \bar{h}_{m}\right) \mathrm{d} x \\
= & \lim _{m \rightarrow \infty} \int_{\mathbb{R}^{2}} \int_{D} \overline{\Phi_{\kappa}(y, x)} h(y) \mathrm{d} y\left(\Delta+\kappa^{2}\right) \bar{h}_{m}+\int_{D} \overline{\Phi_{\kappa}(y, x)} \nabla h(y) \mathrm{d} y \cdot\left(\Delta+\kappa^{2}\right) \nabla \bar{h}_{m} \mathrm{~d} x \\
= & \lim _{m \rightarrow \infty} \int_{\mathbb{R}^{2}}\left(\Delta_{x}+\kappa^{2}\right) \int_{D} \overline{\Phi_{\kappa}(y, x)} h(y) \mathrm{d} y \bar{h}_{m}+\left(\Delta_{x}+\kappa^{2}\right) \int_{D} \overline{\Phi_{\kappa}(y, x)} \nabla h(y) \mathrm{d} y \cdot \nabla \bar{h}_{m} \mathrm{~d} x \\
= & \lim _{m \rightarrow \infty} \int_{\mathbb{R}^{2}}\left(-\mathbb{1}_{D} h\right) \bar{h}_{m}+\left(-\mathbb{1}_{D} \nabla h\right) \cdot \nabla \bar{h}_{m} \mathrm{~d} x \\
= & -\|h\|_{H^{1}(D)} \cdot
\end{aligned}
$$

Combining the latter with (14), we conclude $\|h\|_{H^{1}(D)}=0$ and that $\widehat{S}_{\Phi}(\kappa)$ is injective.

The corresponding density result for $w$ is now immediate with a pull-back argument for the artificial boundary.

Corollary 4. The range of the single layer operator $\widehat{S}_{\Psi}(\kappa)$ from (4) is dense in $\mathcal{A}_{A}(\kappa):=\left\{h \in H^{1}(D): \operatorname{div}(A \nabla h)+\kappa^{2} n h=0\right\}$ for all wave numbers $\kappa>0$.

Proof. Let $w \in \mathcal{A}_{A}(\kappa)$ be arbitrary. By Lemma 1 we know that $w^{A}:=w \circ L^{-1} \in H^{1}\left(D^{A}\right)$ solves $\Delta w^{A}+\kappa^{2} n w^{A}=0$ on $D^{A}$, where the transformed scatterer was defined in (8) and $L(x):=A^{-\frac{1}{2}} x$. Analogue to that we also associate a surrounding artificial boundary by $\Gamma^{A}:=L(\Gamma)$. Then Theorem 3 guarantees the existence of a sequence $\left\{g_{m}^{A}\right\}_{m \in \mathbb{N}}$ such that the functions

$$
w_{m}^{A}\left(x^{A}\right):=\int_{\Gamma^{A}} \Phi_{\kappa}\left(x^{A}, y^{A}\right) g_{m}^{A}\left(y^{A}\right) \mathrm{d} s\left(y^{A}\right), \quad x^{A} \in D^{A}
$$


have the approximation property $w_{m}^{A} \rightarrow w^{A}$ in $H^{1}\left(D^{A}\right)$. Using the transformation formula for curvilinear coordinates, e.g. [13], $w_{m}^{A}$ can be expressed in terms of our integral operator from (4)

$$
\begin{aligned}
& \int_{\Gamma^{A}} \Phi_{\kappa}\left(x^{A}, y^{A}\right) g_{m}^{A}\left(y^{A}\right) \mathrm{d} s\left(y^{A}\right) \\
= & \int_{\Gamma} \Phi_{\kappa}\left(x^{A}, A^{-\frac{1}{2}} y\right) g_{m}^{A}\left(A^{-\frac{1}{2}} y\right)\left|A^{-\frac{1}{2}} \nu(y)\right| \operatorname{det} A^{-\frac{1}{2}} \mathrm{~d} s(y) \\
= & \int_{\Gamma}\left(\operatorname{det} A^{-\frac{1}{2}} \Phi_{\kappa}\left(x^{A}, A^{-\frac{1}{2}} y\right)\right)\left(g_{m}^{A}\left(A^{-\frac{1}{2}} y\right)\left|A^{-\frac{1}{2}} \nu(y)\right|\right) \mathrm{d} s(y) \\
= & \int_{\Gamma} \Psi_{\kappa}\left(A^{\frac{1}{2}} x^{A}, y\right) g_{m}(y) \mathrm{d} s(y),
\end{aligned}
$$

where we set $g_{m}(y):=g_{m}^{A}\left(A^{-\frac{1}{2}} y\right)\left|A^{-\frac{1}{2}} \nu(y)\right|$ for our transformed coefficient functions. Therefore $w_{m}:=w_{m}^{A} \circ L=\widehat{S}_{\Psi}(\kappa) g_{m}$ generates a sequence in the range of $\widehat{S}_{\Psi}(\kappa)$ which approximates $w$ in $H^{1}(D)$ according to

$$
\begin{aligned}
& \left\|w_{m}^{A}-w^{A}\right\|_{H^{1}\left(D^{A}\right)}^{2} \\
= & \int_{D^{A}}\left|w_{m}^{A}-w^{A}\right|^{2} \mathrm{~d} x^{A}+\int_{D^{A}}\left|\nabla w_{m}^{A}-\nabla w^{A}\right|^{2} \mathrm{~d} x^{A} \\
= & \int_{D} \operatorname{det} A^{-\frac{1}{2}}\left|\left(w_{m}^{A}-w^{A}\right) \circ L\right|^{2} \mathrm{~d} x+\int_{D} \operatorname{det} A^{-\frac{1}{2}}\left|\left(\nabla w_{m}^{A}-\nabla w^{A}\right) \circ L\right|^{2} \mathrm{~d} x \\
= & \operatorname{det} A^{-\frac{1}{2}}\left(\int_{D}\left|w_{m}-w\right|^{2} \mathrm{~d} x+\int_{D^{A}}\left|A^{\frac{1}{2}}\left(\nabla w_{m}-\nabla w\right)\right|^{2} \mathrm{~d} x\right) \\
\geq & \operatorname{det} A^{-\frac{1}{2}} \min \left\{1, \sqrt{A_{*}}\right\}\left(\int_{D}\left|w_{m}-w\right|^{2} \mathrm{~d} x+\int_{D^{A}}\left|\left(\nabla w_{m}-\nabla w\right)\right|^{2} \mathrm{~d} x\right) \\
= & \operatorname{det} A^{-\frac{1}{2}} \min \left\{1, \sqrt{A_{*}}\right\} \| w_{m}-w_{H^{1}(D)}^{2} .
\end{aligned}
$$

\section{Numerical results}

In this section, we compute ITEs for a variety of anisotropic scatterers and thereby analyze the effects on the eigenvalues of perturbing the material parameters within the ITP.

\subsection{Computing ITEs with the modified MFS}

In previous papers the challenge to compute concrete ITEs for homogeneous and anisotropic media in 2D were either encountered with pure focus on that purpose, see [20], or as a byproduct of solving the actual inverse electromagnetic scattering problem in 3D under certain symmetry assumptions, see [9] for example. They use boundary element methods and finite element methods, respectively, to discretize the underlying problem whose results we will take as benchmark values to see how competitive the 


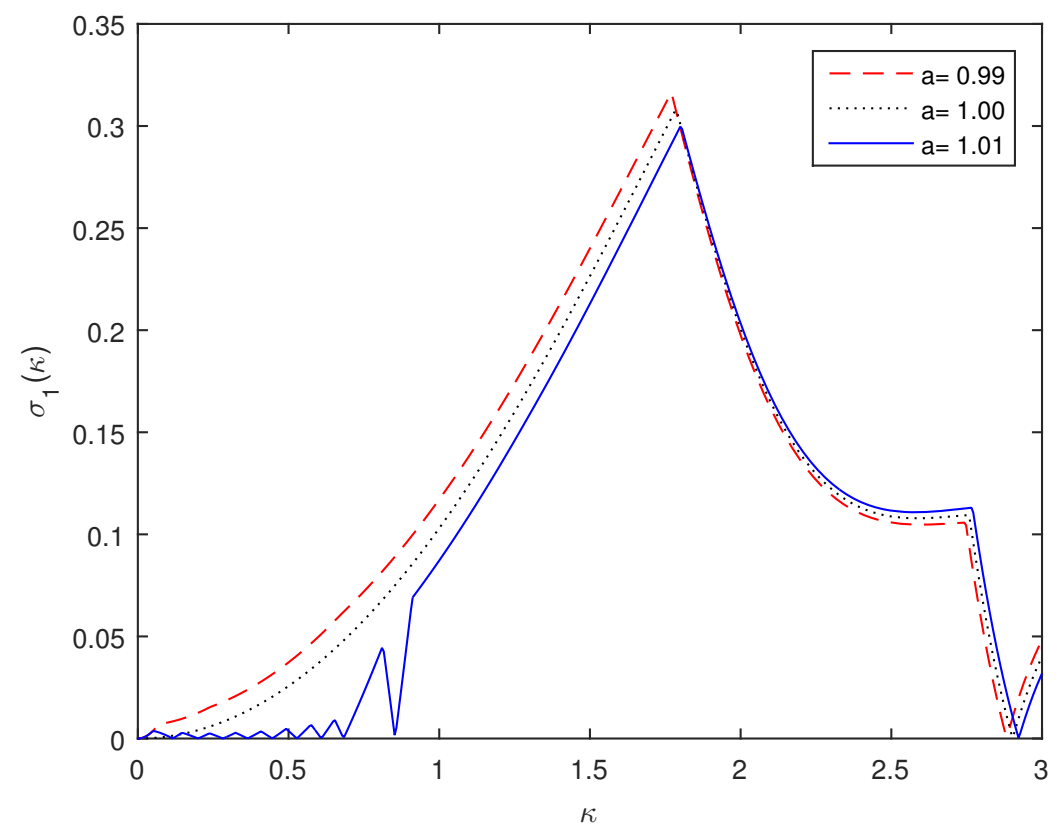

Figure 1. Different ITE behavior for the unit disc $D$ near $\kappa=0$ with parameters $n=4$ and $A^{*}=A_{*}=a$ being slightly larger or smaller than 1.

modified MFS is for the anisotropic ITP. However, we would like to go a little further and focus more closely on the cases $A_{*}>1$ and $A *<1$ which turn out to be also significantly different from a numerical perspective instead of only disjoint auxiliary situations to guarantee the conventional Fredholm setting in theory. To see this, let us fix $n=4$ and assume that $A=\operatorname{diag}(a, a)$ with $a \in\{0.99,1.0,1.01\}$, i.e. we perturb the homogeneous isotropic case with respect to the anisotropic coefficient $a$ slightly both from below and above. Figure 1 was generated for $D$ being the unit disc and for MFS parameter $m=20, m_{I}=10$, whose associated points lie equidistantly on concentric circles inside (radius $=0.5$ ) and outside (radius $=5$ ) of $D$, respectively. As evanescent valleys of the smallest-singular-value-function shall indicate the existence of ITEs according to Theorem 2 and (7) for $m \rightarrow \infty$, these snapshots already show that while the trivial ITE $k=0$ with its infinite dimensional eigenspace for $a=1$ is uniformly isolated if $a<1$, there seems to be an accumulation of eigenvalues around zero for $a>1$, consisting of 9 ones so far. Since the corresponding ITP is rotationally symmetric here, we may even compute the eigenvalues almost analytically using a Fourier-Besselexpansion, see [5]. Accordingly, we need to solve

$$
\operatorname{det}\left(\begin{array}{cc}
J_{p}(\kappa) & J_{p}\left(\sqrt{\frac{n}{a}} \kappa\right) \\
\kappa J_{p}^{\prime}(\kappa) & \sqrt{n a} \kappa J_{p}^{\prime}\left(\sqrt{\frac{n}{a}} \kappa\right)
\end{array}\right)=0,
$$

where $J_{p}$ is the Bessel function of first kind of order $p \in \mathbb{N}$ (note the primes appearing in the bottom line of the matrix denote outer derivatives of the Bessel function) and 


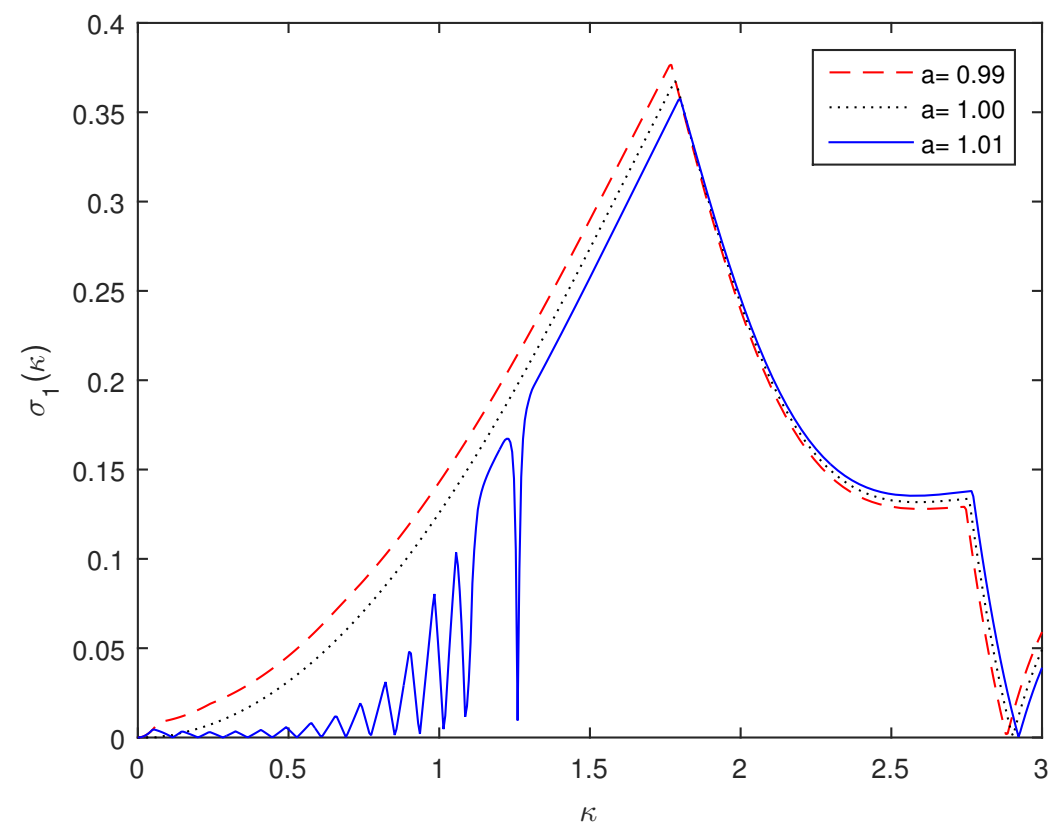

Figure 2. With identical input data as in Figure 1 except for a larger number of collocation points $m$, additional minima come into play for $a=1.01$, while the two graphs for $a \leq 1$ keep rather unchanged.

whose positive roots in $\kappa$ coincide with those of

$$
g_{p}(\kappa, a)=f_{p}(\kappa)-\frac{f_{p}\left(\sqrt{\frac{n}{a}} \kappa\right)}{a},
$$

where

$$
f_{p}(x)=\frac{p J_{p}(x)}{J_{p}^{\prime}(x) x} .
$$

Numerical calculations even confirm for $a=1.01$ the existence of at least 24 ITEs, almost equidistantly distributed, within the interval $(0,2)$ as the consecutive smallest positive roots $\kappa_{p}$ of $g_{p}$ for $p=1, \ldots, 24$ :

$0.115311585535849,0.199588421652397,0.282144281238904,0.364146487789215$,

$0.445898824589272,0.527516720334738,0.609053860429526,0.690538657514437$,

0.771987575480684, 0.853410820048637, 0.934815057189782, 1.016204826695360,

1.097583327960807, 1.178952881037817, 1.260315209516204, 1.341671620743993,

$1.423023124392495,1.504370512663545,1.585714415871614,1.667055341806005$,

$1.748393704134480,1.829729843252073,1.911064041822514,1.992396536525989$.

We now compare these values with each positive root $\widetilde{\kappa}_{p}$ of the second order Taylor approximation $\widetilde{g}_{p}(\cdot, a)$ of $g_{p}(\cdot, a)$ around zero,

$$
\widetilde{g}_{p}(\kappa, a)=\frac{a-1}{a}-\frac{n-a^{2}}{2 a^{2} p(p+1)} \kappa^{2} .
$$


Since $\widetilde{g}_{p}(\cdot, a)$ is strictly decreasing for $a^{2}<n$, but $\widetilde{g}_{p}(0, a)<0$ for $a<1$, we get a first idea about why we do not observe ITEs close to zero for $a=0.99$. However, for $a>1$ the parabola intersects the $\kappa$-axis at

$$
\widetilde{\kappa}_{p}=\sqrt{\frac{2\left(a^{2}-a\right)}{n-a^{2}} p(p+1)},
$$

and evaluates to $0.116436686356126,0.201674256633773,0.285210468912994$ and $1.852038873292762,1.934391514809814,2.016742566337728$ for our marginal test samples $p=1,2,3$ and $p=22,23,24$, respectively. We recognize the almost equidistant structure of the $\kappa_{p}$ consistent with our approximation formula (17) which gives rise to believe that this pattern even continues for larger $p$. Obviously, the modified MFS does not point to all of these existent eigenvalues with a valley closeby yet. Increasing the number of collocation points to $m=30$, we see in Figure 2 that successively further, but still not all minima, especially not those which are more distant from zero, appear within an acceptable number of collocation points. In fact, for larger $m$ the output starts getting negatively accompanied by effects of ill-conditioning which finally results in noisy artefacts within the graphs. We conclude that the regime $a>1$ with $n>1$ (or equivalently $a<1$ with $n<1$ ) seems to be not well suited for eigenvalue approximations on the basis of the modified MFS as long as the indicating minima of the smallestsingular-value-function are forced to oscillate strongly. The following lemma shows that the situation is even worse for $a \searrow 1$, since we have an accumulation of infinitely many ITEs around zero. Our interpretation of this observation is that the eigenspace of $k=0$ for $a=1$, which consists of all harmonic functions, emerges from the collection of eigenstates that are absorbed by the trivial ITE in the right-hand-side limit of $a$.

Lemma 5. Fix $n>1$. Then for any $p \in \mathbb{N}$ and any upper threshold $\widetilde{\kappa}>0$ there exists $\tilde{a}_{p}>1$ such that for all $\widetilde{a}_{p}>a>1$ there exists a positive root $k_{p, a}$ of $g_{p}(\cdot, a)$ from (15) with $\widetilde{\kappa}>k_{p, a}$. The same holds true for the sign relations $n<1$ and $\widetilde{a}_{p}<a<1$ correspondingly.

Proof. We will work with $n, a>1$ since the proof for the converse case can be performed in a similar fashion. One way to prove the lemma is to argue rigorously that (17) sufficiently approximates the smallest root of $g_{p}(\cdot, a)$ asymptotically for $a \searrow 1$. However, we will go a slightly different way and make use of the intermediate value theorem to prove that the continuous function $g_{p}(\cdot, a)$ switches its sign in the interval $(0, \widetilde{\kappa})$ for every $1<a<\widetilde{a}_{p}$. For this we first note that by (16) we have that

$$
g_{p}(0, a)=\frac{a-1}{a}>0
$$

for all $a>1$. Next, we want to show that $g_{p}\left(\kappa_{p}, a\right)<0$ for some $0<\kappa_{p}<\widetilde{\kappa}$ and for every $1<a<\widetilde{a}_{p}$. Since $f_{p}(x)=1+x^{2} /(2 p(p+1))+\mathcal{O}\left(x^{p+4}\right)$ for $x \rightarrow 0$, we can always find an open interval $I_{p}=\left(0, i_{p}\right), i_{p}>0$ on which $f_{p}$ is strictly monotonically increasing. 
For now fix some $1<\widetilde{n}<n$ as well as $\widetilde{\kappa}>\kappa_{p}>0$ such that $\kappa_{p} \sqrt{n}<i_{p}$. Then we set

$$
\widetilde{a}_{p}:=\min \left\{\widetilde{n}, \frac{f_{p}\left(\sqrt{\frac{n}{\tilde{n}}} \kappa_{p}\right)}{f_{p}\left(\kappa_{p}\right)}\right\}>1 .
$$

Hence, for any $1<a<\widetilde{a}_{p}$ we get by monotonicity of $f_{p}$ on $I_{p}$ that

$$
0>f_{p}\left(\kappa_{p}\right)-\frac{f_{p}\left(\sqrt{\frac{n}{\tilde{n}}} \kappa_{p}\right)}{a}>f_{p}\left(\kappa_{p}\right)-\frac{f_{p}\left(\sqrt{\frac{n}{a}} \kappa_{p}\right)}{a}=g_{p}\left(a, \kappa_{p}\right) .
$$

Since $\kappa_{p}$ is independent of $a$, the intermediate value theorem applies in combination with (18) for all $1<a<\widetilde{a}_{p}$ and thereby ensures the existence of roots $k_{p, a}>0$.

However, despite the different behavior of the eigenvalues for $a \nearrow 1$ and $a \searrow 1$ including their retarded numerical appearance for the latter case, it should be noted that Figures 1 and 2 also indicate that for any approximate ITE $k_{-}$such that $a<1$, we can already find very early with respect to the number of collocation points an $(a>1)$-complementing ITE $k_{+}$with the same limit point when the corresponding $a$ approaches 1. Our exemplary reference pair from the right corner of the above plots can even be recovered by the modified MFS up to machine precision and gives the smallest ITE 2.882728798537896 for $a=0.99$ in combination with 2.922641535098038 for $a=1.01$, thus surrounding closely the smallest isotropic ITE of the unit disc being 2.902608055212766, see [21]. In particular, the modified MFS is here far more accurate than the boundary element method from [20] where apparently only 2 digits of their given ITE approximations are correct.

Since the cases $n>1$ and $n<1$ seem to be structurally similar, we now concentrate on $n>1$. Although we have not yet compared numerically the borderline case $n=1$ for both discussed $a$-scenarios, we recall the existence of a scaling law here connecting ITEs for anisotropic reciprocals according to

$$
\begin{array}{rllrl}
a \Delta w+k^{2} w=0 & \text { in } D & & \frac{1}{a} \Delta v+\left(\frac{k}{\sqrt{a}}\right)^{2} v=0 & \text { in } D \\
\Delta v+k^{2} v=0 & \text { in } D \\
w=v & \text { on } \partial D \\
a \partial_{\nu} w=\partial_{\nu} v & \text { on } \partial D & & \Delta w+\left(\frac{k}{\sqrt{a}}\right)^{2} w=0 & \text { in } D \\
v=w & \text { on } \partial D \\
& & \frac{1}{a} \partial_{\nu} v=\partial_{\nu} w & \text { on } \partial D
\end{array}
$$

and which directly links the two separate regimes $a>1$ and $a<1$. Convinced of all this, we leave the guarded zone of analytically computable ITEs via Bessel functions now and provide results for $n \geq 1$ but $A^{*} \neq A_{*}$ in the sequel without having exact reference values at hand any more. However, we still assume $A$ to be diagonal by its tensor property which could be balanced otherwise by a rotation of the scatterer according to

$$
\begin{aligned}
\left.\operatorname{div}(\widetilde{A} \nabla \widetilde{w})\right|_{x} & =\operatorname{tr}\left(\left.\widetilde{A} \nabla \nabla^{T} \widetilde{w}\right|_{x}\right)=\operatorname{tr}\left(\left.\widetilde{A} \nabla \nabla^{T} w\right|_{Q x} Q\right) \\
& =\operatorname{tr}\left(\left.\widetilde{A} Q^{T} \nabla \nabla^{T} w\right|_{Q x} Q\right)=\operatorname{tr}\left(Q\left(\left.\widetilde{A} Q^{T} \nabla \nabla^{T} w\right|_{Q x} Q\right) Q^{T}\right) \\
& =\operatorname{tr}\left(\left.A \nabla \nabla^{T} w\right|_{Q x}\right)=\left.\operatorname{div}(A \nabla w)\right|_{Q x},
\end{aligned}
$$


where we utilized the similarity invariance of the trace and the rotation-associated notation $\widetilde{w}(x):=w(Q x)$ and $\widetilde{A}=Q^{T} A Q$ for $Q \in \mathrm{SO}(2)$. Also, we require that either $A^{*}<1$ or that $A_{*}>1$ is sufficiently distant from unity to avoid for the latter the aforementioned eigenvalue accumulation consequences in practice. Altogether, this results in four different test cases characterized by the constitutive data $\left(A_{11}, A_{22}, n\right)$

\begin{tabular}{l|l} 
Material class & $\left(\boldsymbol{A}_{\mathbf{1 1}}, \boldsymbol{A}_{\mathbf{2 2}}, \boldsymbol{n}\right)$ \\
\hline$\left(A_{*}<1, n=1\right)$ & $\left(\frac{1}{8}, \frac{1}{2}, 1\right)$ \\
$\left(A^{*}>1, n=1\right)$ & $(2,8,1)$ \\
$\left(A_{*}<1, n>1\right)$ & $\left(\frac{1}{8}, \frac{1}{2}, 4\right)$ \\
$\left(A^{*}>1, n>1\right)$ & $(2,8,4)$
\end{tabular}

for which we have sampled the 3-tuple on the right as a class representative to compute the first four real ITEs for. This will be done in the following for a collection of star-shaped scatterers $D$ taken from [21] which are categorized in groups of gradual boundary deformations for better sensitivity control of the corresponding ITE response. Throughout, as the location of interior points did not influence the modified MFS output significantly, we distributed 20 of them in total on circles fully contained in the scattering domain. However, the distribution of the sources, which we restricted in an equiangular arrangement either to the artificial boundary $\Gamma=\partial D(S)$ of the same but slightly scaledup scatterer by some factor $S>1$ (i.e. $\partial D(1)=\partial D$ ) or to some outer circle $\Gamma=\partial B_{R}(0)$ of radius $R$ with its center at the origin, really impacts the approximation quality of the eigenvalues. So our numerical experiments determined quite individually for each $D$ how to choose $S$ or $R$, respectively. As a rule of thumb, the larger $S$ or $R$ is, the faster the MFS output converges with respect to $m$, but simultaneously ill-conditioning effects start earlier to impede the detection. For instance, scatterers of complex shape require more representative collocation points and thus $\Gamma$ should lie closer to $D$ for relatively optimal performance. With these conventions, $m$ (note $m_{I}=20$ is considered as fixed) in combination with $S$ or $R$ fully describes our modified MFS setup for our 2D scattering domains to be discussed, whereas the modeling parameter are given by (20).

We start with the unit disc interpreted as an ellipse with equal axes and shrink the minor axis $b$ step by step to $0.8,0.5$ and 0.3 according to

$$
t \mapsto(\cos (t), b \sin (t)), \quad 0 \leq t<2 \pi
$$

The results are shown in Figure 3 and were obtained for $R=5 b$ as some averaged and cut-off ITE output from those $m$ within the range $\{30, \ldots, 70\}$ whose first singular value were dominantly small. Typical bottom magnitudes were around $10^{-14 \pm 1}$ and sometimes even vanished up to machine precision. We observe that the number of recoverable digits is relatively proportional to the logarithm of $\sigma_{1}$, mostly decreases the more the ellipses deviate from the unit disc and also seems to depend strongly on the material parameters $\left(A_{11}, A_{22}, n\right)$. An explanation for the latter is that the anisotropic parameters enter in our MFS ansatz as inner variations of the fundamental solutions, see Lemma 1, which, in terms of a pull-back, resembles spatial evaluations of our radial basis functions along either more or less elliptic collocation boundaries according to (8). From [21] we even 

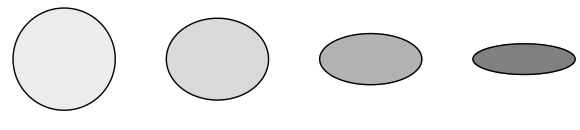

\begin{tabular}{|c|c|c|c|c|c|}
\cline { 3 - 6 } \multicolumn{2}{c|}{} & \multicolumn{2}{c}{$b=1.0-b=0.8-b=0.5-b=0.3$} & \multicolumn{1}{c}{} \\
\hline $\boldsymbol{b}$ & $\left(\boldsymbol{A}_{\mathbf{1 1}}, \boldsymbol{A}_{\mathbf{2 2}}, \boldsymbol{n}\right)$ & ITE 1 & ITE 2 & ITE 3 & ITE 4 \\
\hline \multirow{4}{*}{1.0} & $(1 / 8,1 / 2,1)$ & 2.432342816525 & 2.630469896016 & 3.59558793161 & 3.67772382000 \\
\cline { 2 - 6 } & $(2,8,1)$ & 5.422742998449 & 6.104903895458 & 6.701155193070 & 6.807186612188 \\
\cline { 2 - 6 } & $(1 / 8,1 / 2,4)$ & 1.102569011198 & 1.54251886201 & 1.59628152267 & 1.60949290139 \\
\cline { 2 - 6 } & $(2,8,4)$ & 1.0943753002121 & 2.6607655055967 & 3.2850748911309 & 4.741801624682 \\
\hline \multirow{4}{*}{0.8} & $(1 / 8,1 / 2,1)$ & 2.8165335886620 & 2.9285919681719 & 4.07689045702 & 4.29727034976 \\
\cline { 2 - 6 } & $(2,8,1)$ & 6.533520997906 & 7.416596066665 & 7.518127550666 & 7.698364095496 \\
\cline { 2 - 6 } & $(1 / 8,1 / 2,4)$ & 1.2746345105108 & 1.2937710253833 & 1.708033787158 & 1.764558638514 \\
\cline { 2 - 6 } 0.5 & $(2,8,4)$ & 1.0949963906964 & 3.295035558059 & 3.883765024644 & 4.66548759617 \\
\cline { 2 - 6 } & $(1 / 8,1 / 2,1)$ & 4.04671357026 & 4.0805282756 & 5.2097578799 & 5.2372873746 \\
\cline { 2 - 6 } & $(2,8,1)$ & 10.014688061884 & 10.43413680087 & 10.67428849705 & 11.00440368778 \\
\cline { 2 - 6 } & $(1 / 8,1 / 2,4)$ & 1.86432077115 & 1.86788372558 & 2.2749471210 & 2.2899879956 \\
\hline \multirow{4}{*}{0.3} & $(2,8,4)$ & 1.094252956180 & 2.153141477577 & 3.263881703236 & 3.587938159800 \\
\cline { 2 - 6 } & $(1 / 8,1 / 2,1)$ & 6.3010969 & 6.3020803 & 7.352124 & 7.361319 \\
\cline { 2 - 6 } & $(2,8,1)$ & 16.1836653485 & 16.2620312417 & 16.317697464 & 16.50554137 \\
\cline { 2 - 6 } & $(1 / 8,1 / 2,4)$ & 2.94403372897 & 2.94412360374 & 3.338511006 & 3.338761987 \\
\hline
\end{tabular}

Figure 3. First four approximate real-valued ITEs without counting multiplicity for ellipses with major semi-axis of length 1 and varied minor semi axis $b$ based on the modified MFS.

know that the best results were achieved for the disc among all scattering ellipses under consideration and so it is actually not surprising that $A_{11}=A_{22}$ still yields superior accuracy.

Next, we focus on the transition from an ellipse to a kite shape whose parametrization

$$
t \mapsto(0.75 \cos (t)+d \cos (2 t), \sin (t)), \quad 0 \leq t<2 \pi
$$

is originally taken from [11]. Here, $d$ is the characterizing deformation parameter which we will increase successively from 0 to 0.3 by steps of size 0.1 . Figure 4 displays our MFS results for this case and shows in comparison with Figure 3 that due to the more complex scattering shapes we have now lost accuracy altogether. In particular, the larger the perturbation $d$ is, the smaller the extracted approximation mantissa is. This regression was also indicated by the magnitudes of $\sigma_{1}$ during the computational procedure which were roughly bounded from below by $10^{-15+20 d}$ each. It became necessary to choose a tighter scaling factor for $\Gamma$ that turned out to be numerically quite optimal for $S=2-d$. Likewise, the number of collocation points required for some stable MFS output was slightly larger than for the ellipses and ranged from about 35 to 75 . As an intermediate conclusion, the modified MFS seems very powerful for rather easy profiles of $D$ and begins to stagnate gradually as one deviates from the isotropic case or from the disc as scatterer.

In order to see how the method responds to shapes with corners and in particular to Lipschitz domains, we examine the unit square $D=[-0.5,0.5]^{2}$. It is clear at first sight 


\begin{tabular}{|c|c|c|c|c|c|}
\hline & & $d=0.0$ & $d=0.1 \longrightarrow d=0$. & $-d=0.3$ & \\
\hline$d$ & $\left(A_{11}, A_{22}, n\right)$ & ITE 1 & ITE 2 & ITE 3 & ITE 4 \\
\hline \multirow{4}{*}{0.0} & $(1 / 8,1 / 2,1)$ & 2.8471025260348 & 3.1600761111251 & 3.8992194907200 & 4.0633832879825 \\
\hline & $(2,8,1)$ & 5.813165368996797 & 6.483618475707658 & 7.70907468441514 & 8.0359270276980 \\
\hline & $(1 / 8,1 / 2,4)$ & 1.25548301224033 & 1.25437465935063 & 1.69500812538264 & 1.75549467161328 \\
\hline & $(2,8,4)$ & 1.45615282653635 & 2.7053687255745 & 3.5755403402386 & 5.3469141120703 \\
\hline \multirow{4}{*}{0.1} & $(1 / 8,1 / 2,1)$ & 2.844832741265 & 3.175258583036 & 3.894779056630 & 4.09507454531 \\
\hline & $(2,8,1)$ & 5.849387560813 & 6.477010844996 & 7.728576868915 & 7.99190658583 \\
\hline & $(1 / 8,1 / 2,4)$ & 1.234632318588 & 1.257786433541 & 1.699972423624 & 1.762508594273 \\
\hline & $(2,8,4)$ & 1.438716788003 & 2.694965222759 & 3.55348405570 & 5.395815566 \\
\hline \multirow{4}{*}{0.2} & $(1 / 8,1 / 2,1)$ & 2.868977355 & 3.19024736 & 3.929439900 & 4.14548893 \\
\hline & $(2,8,1)$ & 5.96482555 & 6.48350477 & 7.805845131 & 7.91285541 \\
\hline & $(1 / 8,1 / 2,4)$ & 1.255163101 & 1.273766679 & 1.714003852 & 1.788830942 \\
\hline & $(2,8,4)$ & 1.392899663 & 2.643884081 & 3.493341629 & 5.62747817 \\
\hline \multirow{4}{*}{0.3} & $(1 / 8,1 / 2,1)$ & 2.93627 & 3.2048 & 4.03421 & 4.1863 \\
\hline & $(2,8,1)$ & 6.1597 & 6.5494 & 7.8795 & 7.9617 \\
\hline & $(1 / 8,1 / 2,4)$ & 1.27293 & 1.31188 & 1.73880 & 1.83879 \\
\hline & $(2,8,4)$ & 1.32210 & 2.49773 & 3.42292 & 4.28398 \\
\hline
\end{tabular}

Figure 4. First four approximate real-valued ITEs without counting multiplicity for the shape transition ellipse-kite with deformation parameter $d$ based on the modified MFS.

\begin{tabular}{|c|c|c|c|c|}
\hline$\left(\boldsymbol{A}_{\mathbf{1 1}}, \boldsymbol{A}_{\mathbf{2 2}}, \boldsymbol{n}\right)$ & ITE 1 & ITE 2 & ITE 3 & ITE 4 \\
\hline$(1 / 8,1 / 2,1)$ & 4.386 & 4.845 & 6.615 & 6.646 \\
\hline$(2,8,1)$ & 10.036 & 11.14 & 11.60 & 12.32 \\
\hline$(1 / 8,1 / 2,4)$ & 1.998 & 2.021 & 2.798 & 2.853 \\
\hline$(2,8,4)$ & 1.85 & 4.92 & 5.14 & 6.11 \\
\hline
\end{tabular}

Figure 5. First four approximate real-valued ITEs without counting multiplicity based on the modified MFS for the unit square.

in Figure 5 that the modified MFS lacks accuracy here despite the high symmetry of the scatterer. We recall from the isotropic case, considered as the optimal MFS scenario, that there is the deep result from [4] which states that solution pairs $(v, w)$ of the ITP cannot be extended locally around right angle corners. In this spirit, we get an idea about why we were forced to take $S=1.2$ which is very close to $D$ itself to find reliable ITE results. However, we are still able to improve the unit square results from [9] for the smallest ITE induced by the material parameters $\left(A_{11}, A_{22}, n\right)=(1 / 8,1 / 2,1)$ which was predicted as 4.1 there and updated now as 4.386 . In our calculation process, $m$ often needed to exceed 100 collocation points until minimal dips for $\sigma_{1}$ in the graph became effectively apparent. We conclude that, especially for non-smooth scattering boundaries, the MFS rather serves as a rough ITE localizer than as an accuracy gainer.

We complete this numerical section by pointing out that the modified MFS has still more ITE features to offer. For example, the method is optionally capable of recovering the multiplicity of each approximate eigenvalue by repeating the whole algorithm with 
the first singular value in (6) replaced by higher ones: if, in the notation from Theorem $2, k_{m} \rightarrow k, \sigma_{1}\left(k_{m}\right)$ vanishes for $m \rightarrow \infty$ asymptotically like $\sigma_{2}\left(k_{m}\right), \ldots, \sigma_{i}\left(k_{m}\right)$ for some fixed $2 \leq i \in \mathbb{N}$, then we have a first indication that the multiplicity of $k$ as an ITE might also be $i$. Such an adoption for the limit certainly requires additional justification and advanced assumptions on the eigenfunctions. However, unlike other eigenvalue algorithms, for each $m$ our method manages to separate the clustering of closely nearby and possibly simple eigenvalues from identical ones having a corresponding multiplicity. Also, there is the possibility of recovering the approximate ITE eigenfunctions for every $k_{m}$ in terms of $\sigma_{1}\left(k_{m}\right)=\cdots=\sigma_{i}\left(k_{m}\right)$ and their corresponding singular vectors. Again one needs to adapt our current solution algorithm slightly and invoke the so called generalized SVD instead of (6) to circumvent severe ill-conditioning problems, see [1].

For more insights or further details of the MFS in the ITE context, we refer to [21].

\subsection{Conclusion}

The modified MFS is an easy but powerful tool for anisotropic ITE recovery dealing with relatively easy shapes such that a moderate number of collocation points is sufficient to represent the scatterer's boundary. To obtain optimal results in terms of the approximation quality, a key step is finding a proper exterior artificial boundary unique to the scatterer itself whose (non-linear) optimization we did not focus on yet. Instead, our experiments with star-shaped domains has proven to be very competitive with a copy of the positively scaled-up scattering boundary or with a simple surrounding circle for the source points. Working with more exotic domains or with Lipschitz domains, the method lacks higher accuracy, but is still capable of detecting the rough locality of ITEs. For the first time, the two ordered regimes of $(A-I)$ around the isotropic case were investigated with a focus on the corresponding distribution of the ITEs. Our numerical results show that they behave significantly differently which limits the applicability of our method close to certain borderline cases of $A$. It would be interesting to know whether finite element methods are also affected by this problem. In our future research, we try to tackle a convergence analysis for the modified MFS in the ITP context to have a more rigorous measure at hand for the method's efficiency.

\section{Acknowledgments}

The corresponding author thanks Prof. Andreas Kirsch for his inspiring suggestions and discussion on the topic of this paper during his visit in Karlsruhe.

\section{References}

[1] T. Betcke. The generalized singular value decomposition and the method of particular solutions. SIAM Journal on Scientific Computing, 30(3):1278-1295, 2008.

[2] T. Betcke and L. N. Trefethen. Reviving the method of particular solutions. SIAM Review, 47(3):469-491, 2005. 
[3] E. Blåsten, X. Li, H. Liu, and Y. Wang. On vanishing and localizing of transmission eigenfunctions near singular points: a numerical study. Inverse Problems, 33(10):105001, 2017.

[4] E. Blåsten, L. Päivärinta, and J. Sylvester. Corners always scatter. Communications in Mathematical Physics, 331:725-753, 2014.

[5] F. Cakoni and D. Colton. A Qualitative Approach to Inverse Scattering Theory. Springer Science \& Business Media, Heidelberg, 2013.

[6] F. Cakoni, D. Colton, and H. Haddar. The linear sampling method for anisotropic media. Journal of Computational and Applied Mathematics, 146(2):285-299, 2002.

[7] F. Cakoni, D. Colton, and H. Haddar. The computation of lower bounds for the norm of the index of refraction in an anisotropic media from far field data. The Journal of Integral Equations and Applications, 21(2):203-227, 2009.

[8] F. Cakoni, D. Colton, and H. Haddar. Inverse Scattering Theory and Transmission Eigenvalues. SIAM, Philadelphia, 2016.

[9] F. Cakoni, D. Colton, P. Monk, and J. Sun. The inverse electromagnetic scattering problem for anisotropic media. Inverse Problems, 26(7):074004, 2010.

[10] F. Cakoni and H. Haddar. Transmission eigenvalues in inverse scattering theory. Inside Out II, 60:527-578, 2012.

[11] F. Cakoni and R. Kress. A boundary integral equation method for the transmission eigenvalue problem. Applicable Analysis, 96(1):23-38, 2017.

[12] D. Colton, P. Monk, and J. Sun. Analytical and computational methods for transmission eigenvalues. Inverse Problems, 26(4):045011, 2010.

[13] M. E. Gurtin. An Introduction to Continuum Mechanics, volume 158. Academic Press, New York, 1982.

[14] I. Harris, F. Cakoni, and J. Sun. Transmission eigenvalues and non-destructive testing of anisotropic magnetic materials with voids. Inverse Problems, 30(3):035016, 2014.

[15] G. C. Hsiao, F. Liu, J. Sun, and L. Xu. A coupled BEM and FEM for the interior transmission problem in acoustics. Journal of Computational and Applied Mathematics, 235(17):5213-5221, 2011.

[16] X. Ji and J. Sun. A multi-level method for transmission eigenvalues of anisotropic media. Journal of Computational Physics, 255:422-435, 2013.

[17] A. Kirsch. Factorization of the far-field operator for the inhomogeneous medium case and an application in inverse scattering theory. Inverse Problems, 15(2):413-429, 1999.

[18] A. Kirsch. An integral equation for the scattering problem for an anisotropic medium and the factorization method. In Advanced Topics in Scattering and Biomedical Engineering, pages 57-70. World Scientific, 2008.

[19] A. Kirsch and N. Grinberg. The Factorization Method for Inverse Problems. Oxford University Press, Oxford, 2008.

[20] A. Kleefeld and D. Colton. Interior transmission eigenvalues for anisotropic media. In C. Constanda, M. Dalla Riva, P. Lamberti, and P. Musolino, editors, Integral Methods in Science and Engineering, volume 1, pages 139-147. Springer, 2017.

[21] A. Kleefeld and L. Pieronek. The method of fundamental solutions for computing acoustic interior transmission eigenvalues. Inverse Problems, 34(3):035007, 2018.

[22] E. Lakshtanov and B. Vainberg. Ellipticity in the interior transmission problem in anisotropic media. SIAM Journal on Mathematical Analysis, 44(2):1165-1174, 2012.

[23] A. Lechleiter and S. Peters. Determining transmission eigenvalues of anisotropic inhomogeneous media from far field data. Commun. Math. Sci, 13(7):1803-1827, 2015. 